\title{
Research on Static Decoupling Method of Non-Gyro Micro Inertial Measurement Unit
}

\author{
Mingli Ding, Dongmei Yang, Jindong Zhao, and Lili Zhuang \\ Dept. of Automatic Test and Control, Harbin Institute of Technology, \\ 150001 Harbin, China \\ dingmingli2006@gmail.com
}

\begin{abstract}
In a non-gyro micro inertial measurement unit (NGMIMU), the coupling error reduces the system measurement precision obviously. Based on the definition of the coupling error, a new static decoupling method applied total least squares (TLS) algorithm is proposed. TLS considers not only the error of accelerometer output, but also the calibrating error of the input signal arising from the couple factor. Based on TLS, the solution of the coupling coefficient equation has the characteristic of minimum norm to the input and output values. In the navigation parameter estimation, according to the relationship of the accelerometer input and output value, the accurate estimated input value can be acquired through fitting the couple coefficient matrix using TLS and reconstructing the input value using Moore-Penrose generalized inverse matrix. A simulation case for estimating angle rate is investigated by this approach. The results show that the ratio of decoupling error is less than $8 \%$ and verify the feasibility of the static decoupling method.
\end{abstract}

Keywords: Non-gyro micro measurement unit (NGMIMU), decoupling, total least squares (TLS).

\section{$1 \quad$ Introduction}

Most current micro inertial measurement units (MIMU) use linear accelerometers and gyroscopes to sense linear the acceleration and angular rate of a moving body respectively. In a non-gyro micro inertial measurement unit (NGMIMU)[1-6], accelerometers are not only used to acquire the linear acceleration, but also replace gyroscopes to compute the angular rate according to their positions in three-dimension space. NGMIMU has the advantages of anti-high g value shock, low power consumption, small volume and low cost. It can be applied to some specific occasions such as tactic missiles, intelligent bombs and so on.

A generalized definition of couple is that the response of an actuation includes the additional information about the other actuation which would not affect the response. In a NGMIMU system, the couple defines that the output of a single-axis accelerometer contains the additional information of the other accelerometer in other direction. 
The static couple indicates that the coupling degree is fixed whether the measuring time and measuring conditions change or not. We use the coupling coefficient to denote the coupling degree. The static couple mainly arises from the following reasons: a. the sensing axis of the accelerometer produces a deviation due to the low precision of the accelerometer itself; $b$. the low machining precision of the rigid body which bears the weigh of the accelerometer; c. the low mounting precision of the accelerometer; $d$. it is difficult to realize in physics that all the accelerometers mount at one point on the rigid body when some algorithms are expected. When the accelerometer coupling error is small, the measurement value of the angular rate will distort seriously. So the study of the decoupling method is expected urgently. The key problem of the static decoupling method is to estimate the coupling coefficient. The traditional least squares algorithm could not acquire the ideal estimation precision for it considers only the output error of the accelerometer.

In this paper, Total Least Squares algorithm-TLS[7-9] is applied in the novel static decoupling method. This method reconstructs the linear calibrating input signal under the condition that there exist errors not only in the input signal but also in the output signal. The signal reconstructing has two steps: step 1. fitting the couple coefficient matrix by using TLS, step 2 . reconstructing the calibrating signal by using MoorePenrose generalized inverse matrix.

\section{Accelerometer Output Equation}

As all know, the precession of gyroscopes can be used to measure the angular rate. Based on this principle, MIMU measures the angular rate of a moving body. The angle value can be obtained by integrating the angular rate with given initial conditions. With this angle value and the linear acceleration values in three directions, the current posture of the moving body can be estimated.

NGMIMU uses linear accelerometers to measure the angular acceleration of the body, and the relationship between the linear acceleration $a$ and the angular acceleration $\dot{\omega}$ is

$$
a=r \times \dot{\omega}
$$

The angular rate in a certain direction can be calculated by using the linear acceleration between two points. To obtain the linear and angular motion parameters of a moving body in three-dimension space, the accelerometers need to be appropriately distributed on the moving body and the analysis of the accelerometer outputs is needed.

An inertial frame and a rotating moving body frame are exhibited in Fig. 1, where $b$ represents the moving body frame and $I$ the inertial frame. An inertial frame and a rotating moving body frame are exhibited in Fig. 1, where $b$ represents the moving body frame and $I$ the inertial frame. 


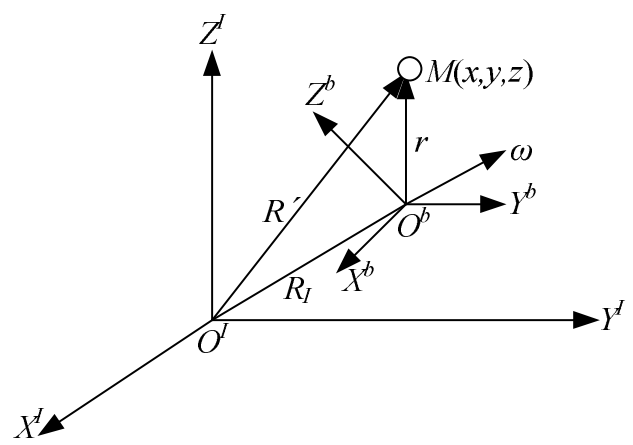

Fig. 1. Geometry of body frame (b) and inertial frame (I)

The acceleration of point $M$ is given by

$$
a=\ddot{R}_{I}+\ddot{r}_{b}+\dot{\omega} \times r+2 \omega \times \dot{r}_{b}+\omega \times(\omega \times r),
$$

where $\ddot{r}_{b}$ is the acceleration of point $M$ relative to body frame. $\ddot{R}_{I}$ is the inertial acceleration of $O^{b}$ relative to $O^{I} .2 \omega \times \dot{r}_{b}$ is known as the Coriolis acceleration, $\omega \times(\omega \times r)$ represents a centripetal acceleration, and $\dot{\omega} \times r$ is the tangential acceleration owing to angular acceleration of the rotating frame.

If $M$ is fixed in the $b$ frame, the terms $\dot{r}_{b}$ and $\ddot{r}_{b}$ vanish. And (2) can be rewritten as

$$
a=\ddot{R}_{I}+\dot{\omega} \times r+\omega \times(\omega \times r) .
$$

Thus the accelerometers rigidly mounted at location $r_{i}$ on the body with sensing direction $\theta_{i}$ produce $A_{i}$ as outputs.

$$
A_{i}=\left[\ddot{R}_{I}+\dot{\Omega}_{i}+\Omega \Omega r_{i}\right] \cdot \theta_{i} \quad(i=1,2, \ldots, N),
$$

where

$$
\Omega=\left[\begin{array}{ccc}
0 & -\omega_{z} & \omega_{y} \\
\omega_{z} & 0 & -\omega_{x} \\
-\omega_{y} & \omega_{x} & 0
\end{array}\right], \quad \ddot{R}_{I}=\left[\begin{array}{c}
\ddot{R}_{I x} \\
\ddot{R}_{I y} \\
\ddot{R}_{I z}
\end{array}\right] .
$$

In (5), $\omega_{x}, \omega_{y}$ and $\omega_{z}$ represent the angular rate along $\mathrm{x}, \mathrm{y}$ and $\mathrm{z}$ axis respectively; $\ddot{R}_{I X}, \ddot{R}_{I y}$ and $\ddot{R}_{I y}$ represent the linear acceleration along $\mathrm{x}, \mathrm{y}$ and $\mathrm{z}$ axis respectively.

Considering $N$ accelerometer distributed at locations $r_{1}, \ldots, r_{N}$ with sensing directions $\theta_{1}, \ldots, \theta_{N}$ respectively. The pair $\left(r_{i}, \theta_{i}\right)$ is express in the body frame. 
Let $\omega=\left[\begin{array}{lll}\omega_{x} & \omega_{y} & \omega_{z}\end{array}\right]^{T}$. Considering the skew-symmetric vector $\Omega$, for any $N=\left[\begin{array}{lll}n_{x} & n_{y} & n_{z}\end{array}\right]^{r}$, we have

$$
\Omega \cdot N=\left[\begin{array}{ccc}
0 & -\omega_{z} & \omega_{y} \\
\omega_{z} & 0 & -\omega_{x} \\
-\omega_{y} & \omega_{x} & 0
\end{array}\right]\left[\begin{array}{l}
n_{x} \\
n_{y} \\
n_{z}
\end{array}\right]=\left[\begin{array}{c}
\omega_{y} n_{z}-\omega_{z} n_{y} \\
\omega_{z} n_{x}-\omega_{x} n_{z} \\
\omega_{x} n_{y}-\omega_{y} n_{x}
\end{array}\right]=\omega \times N .
$$

Using (4) and $\omega \leftrightarrow \Omega$, we have

$$
\begin{aligned}
A_{i} & =\left(\ddot{R}_{I}+\dot{\Omega} r_{i}+\Omega \Omega r_{i}\right) \cdot \theta_{i} \\
& =\theta_{i}^{T} \ddot{R}_{I}+\left(r_{i} \times \theta_{i}\right)^{T} \dot{\omega}+\theta_{i}^{T} \Omega^{2} r_{i} \\
& \left.=\left[\begin{array}{ll}
\left(r_{i} \times \theta_{i}\right)^{T} & \theta_{i}^{T}
\end{array}\right] \begin{array}{c}
\dot{\omega} \\
\ddot{R}_{I}
\end{array}\right]+\theta_{i}^{T} \Omega^{2} r_{i} .
\end{aligned}
$$

\section{Analysis of Static Couple of NGMIMU}

\subsection{Nine-Accelerometer Configuration}

The study of the decoupling method is based on the nine-accelerometer configuration of NGMIMU in [6]. The locations and the sensing directions of the nine accelerometers in the body frame are shown in Fig.2. Each arrow in Fig. 2 points to the sensing direction of each accelerometer.

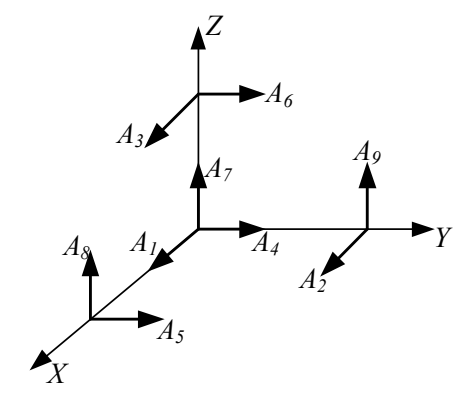

Fig. 2. Nine-accelerometer NGMIMU configuration

The locations of the nine accelerometers are

$$
\left[r_{1}, \cdots, r_{9}\right]=l\left[\begin{array}{lllllllll}
0 & 0 & 0 & 0 & 1 & 0 & 0 & 1 & 0 \\
0 & 1 & 0 & 0 & 0 & 0 & 0 & 0 & 1 \\
0 & 0 & 1 & 0 & 0 & 1 & 0 & 0 & 0
\end{array}\right]
$$


where $l$ is the distance between the accelerometer and the origin of the body frame. The sensing directions are

$$
\left[\theta_{1}, \cdots, \theta_{9}\right]=\left[\begin{array}{lllllllll}
1 & 1 & 1 & 0 & 0 & 0 & 0 & 0 & 0 \\
0 & 0 & 0 & 1 & 1 & 1 & 0 & 0 & 0 \\
0 & 0 & 0 & 0 & 0 & 0 & 1 & 1 & 1
\end{array}\right] .
$$

It is easy to obtain

$$
\left[r_{1} \times \theta_{1}, \cdots, r_{1} \times \theta_{1}\right]=l\left[\begin{array}{ccccccccc}
0 & 0 & 0 & 0 & 0 & -1 & 0 & 0 & 1 \\
0 & 0 & 1 & 0 & 0 & 0 & 0 & -1 & 0 \\
0 & -1 & 0 & 0 & 1 & 0 & 0 & 0 & 0
\end{array}\right] .
$$

Using (7), the accelerometer output equation can be acquired as

$$
A_{i}=\left[\begin{array}{cccccc}
0 & 0 & 0 & 1 & 0 & 0 \\
0 & 0 & -l & 1 & 0 & 0 \\
0 & l & 0 & 1 & 0 & 0 \\
0 & 0 & 0 & 0 & 1 & 0 \\
0 & 0 & l & 0 & 1 & 0 \\
-l & 0 & 0 & 0 & 1 & 0 \\
0 & 0 & 0 & 0 & 0 & 1 \\
0 & -l & 0 & 0 & 0 & 1 \\
l & 0 & 0 & 0 & 0 & 1
\end{array}\right]\left[\begin{array}{c}
\dot{\omega}_{x} \\
\dot{\omega}_{y} \\
\dot{\omega}_{z} \\
\ddot{R}_{l x} \\
\ddot{R}_{l y} \\
\ddot{R}_{I z}
\end{array}\right]+\left[\begin{array}{cccccc}
0 & 0 & 0 & 0 & 0 & 0 \\
0 & 0 & 0 & 0 & 0 & l \\
0 & 0 & 0 & 0 & l & 0 \\
0 & 0 & 0 & 0 & 0 & 0 \\
0 & 0 & 0 & 0 & 0 & l \\
0 & 0 & 0 & l & 0 & 0 \\
0 & 0 & 0 & 0 & 0 & 0 \\
0 & 0 & 0 & 0 & l & 0 \\
0 & 0 & 0 & l & 0 & 0
\end{array}\right]\left[\begin{array}{c}
\omega_{x}^{2} \\
\omega_{y}^{2} \\
\omega_{z}^{2} \\
\omega_{y} \omega_{z} \\
\omega_{x} \omega_{z} \\
\omega_{x} \omega_{y}
\end{array}\right] .
$$

With (11), the linear expressions are

$$
\begin{gathered}
\dot{\omega}_{x}=\frac{1}{2 l}\left(A_{4}+A_{9}-A_{6}-A_{7}\right), \\
\dot{\omega}_{y}=\frac{1}{2 l}\left(A_{3}+A_{7}-A_{1}-A_{8}\right), \\
\dot{\omega}_{z}=\frac{1}{2 l}\left(A_{1}+A_{5}-A_{2}-A_{4}\right),
\end{gathered}
$$

\subsection{Analysis of Static Coupling Error}

In (12), the angular accelerations are all expressed as the linear combinations of the accelerometer outputs. The conventional algorithm computes the angular rate as the time integration of the equations in (12), and gets the rotation matrix of the body frame with respect to earth frame by using a numerical solution, then calculates the posture parameters of the moving body by using the relationship of the elements in the rotation matrix. 
According to the definition of the couple and the reasons for the static coupling error, the static couple of the accelerometer can be regarded as the situation that there is a small angle between the sensing direction of the accelerometer and the body coordinate axis as shown in Fig. 3.

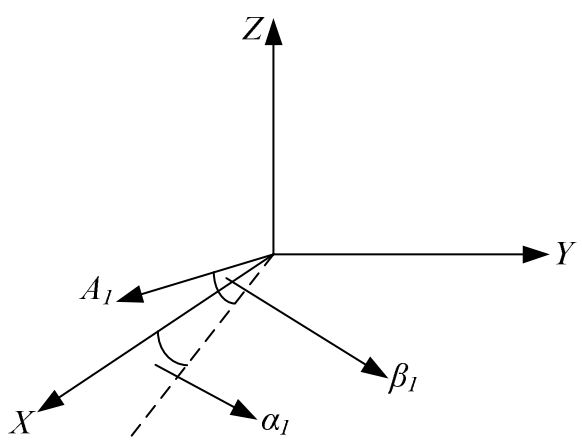

Fig. 3. Real location of accelerometer

For being simple and convenient, the cross coupling problem of $A_{1}, A_{4}$ and $A_{7}$ is specially discussed here. In an idea case, $A_{1}, A_{4}$ and $A_{7}$ are the three accelerometer outputs which are perpendicular each other in the configuration. But due to the static coupling error, the outputs of the accelerometer $A_{1}$ includes the additional acceleration information from the other two accelerometer $A_{4}, A_{7} . A_{4}$ and $A_{7}$ have the similar case. Obviously the computed angular rate has the coupling error and will bring the posture error to the navigation parameters. Assume that the angles between $A_{1}, A_{4}, A_{7}$ and the horizontal direction of the coordinate axis where the corresponding accelerometer locates are $\alpha_{1}, \alpha_{4}, \alpha_{7}$, respectively, the altitude angles are $\beta_{1}, \beta_{4}, \beta_{7}$, respectively. The real accelerometers outputs are

$$
\begin{aligned}
& A_{1}=A_{x} \cos \alpha_{1} \cos \beta_{1}+A_{y} \sin \alpha_{1} \cos \beta_{1}+A_{z} \sin \beta_{1}+C_{1}, \\
& A_{4}=A_{x} \sin \beta_{2}+A_{y} \cos \alpha_{2} \cos \beta_{2}+A_{z} \sin \alpha_{2} \cos \beta_{2}+C_{2}, \\
& A_{7}=A_{x} \sin \alpha_{3} \cos \beta_{3}+A_{y} \sin \beta_{3}+A_{z} \cos \alpha_{3} \cos \beta_{3}+C_{3} .
\end{aligned}
$$

where $A_{x}, A_{y}, A_{z}$ are the real accelerations in three directions. In the course of decoupling, we replace them by the setting input signal calibrated, which all include the calibrating errors. In (13), $C_{1}, C_{2}, C_{3}$ are the static zero drift errors of the accelerometers respectively. From (13a), we arrive that $A_{1}$ includes two real output components of the two accelerometers which are perpendicular with $\mathrm{x}$ axial, and $A_{4}$ and $A_{7}$ have the similar outputs. Based above, it is found that there exists errors not 
only in the calibrating values of the accelerometers $A_{x}, A_{y}, A_{z}$, but also in the accelerometers outputs $A_{1}, A_{4}, A_{7}$. In order to increase the estimation precision of the NGMIMU system, the decoupling method to the corresponding coupling error should be carried on.

\section{Static Decoupling Method}

\subsection{Total-Least Squares Algorithm-TLS}

Consider the equation

$$
A x=b,
$$

where $A \in R^{m \times n}, x \in R^{n}, b \in R^{m}, m \geq n$. Calculating (14) by using the Least Squares algorithm is equivalent to seek the solution $x_{L S}$ which fulfills $\|e\|_{2}=\left\|b-A x_{L S}\right\|_{2}=\min _{x}\|b-A x\|_{2}$. It is obvious that the calculation is based on the assumption that the vector $b$ has a deviation $\Delta b$ and the coefficient matrix $A$ has no deviation, (14) becomes

$$
A x=b+\Delta b .
$$

But in fact, $A$ is often formed by the input signal and the output signal of the system which inevitably have errors. So the best prediction method is to consider the deviations both in $A$ and $b$. This is the key point of the TLS.

Assume $A$ and $b$ have the deviation errors $E$ and $e$ at the same time, (14) can be rewritten as

$$
(A+E) x=b+e .
$$

Now we calculate (16) by using TLS. Change (16) into

$$
(B+D) z=0 \text {, }
$$

where $B=[-b: A], D=[-e: E], z=\left[\begin{array}{l}1 \\ x\end{array}\right]$. So the solution of (17) can be express as to find a vector $z$ such that

$$
\|D\|_{2}=\min ,(b+e) \in \operatorname{range}(A+E),
$$

where $\|D\|_{2}=\left(\sum_{i=1}^{m} \sum_{j=1}^{n} d_{i j}^{2}\right)^{\frac{1}{2}}$. And to the given function (17), TLS can find a vector $x_{T L S}$ such as the following consistency equation

$$
(b+e) \in \operatorname{range}(A+E),
$$


where $\hat{A}$ and $b_{T L S}$ are defined by the optimizing problem which is express as $\min _{x}\left\|[A, b]-\left[\hat{A}, b_{T L S}\right]\right\|_{2}$. It is obvious that the TLS solution has the minimum norm with respect to $A$ and $b$ simultaneously.

Let singular value decomposition (SVD) of matrix $B$ be $B=U S V^{*}$, where $U$ is an unitary matrix with size $m \times m, V$ is an unitary matrix with size $n \times n, V^{*}$ is the associate matrix of $V, S$ is an diagonal matrix with size $m \times n$ and the elements in its leading diagonal is non-negative.

\subsection{Analysis of the Decoupling Method}

Basing the analysis in 3.2, (13) can be expressed by

$$
H \cdot A_{\text {IN }}=A_{\text {out }},
$$

where $H$ is the coupling coefficient matrix and its value is $\left[\begin{array}{llll}\cos \alpha_{1} \cos \beta_{1} & \sin \alpha_{1} \cos \beta_{1} & \sin \beta_{1} & C_{1}\end{array}\right]$ $\left[\begin{array}{cccc}\sin \beta_{2} & \cos \alpha_{2} \cos \beta_{2} & \sin \alpha_{2} \cos \beta_{2} & C_{2} \\ \sin \alpha_{3} \cos \beta_{3} & \sin \beta_{3} & \cos \alpha_{3} \cos \beta_{3} & C_{3}\end{array}\right], A_{I N}$ is the calibrating matrix of the accelerometer input and is express by $\left[\begin{array}{llll}A_{x} & A_{y} & A_{z} & 1\end{array}\right]^{T}, A_{\text {out }}$ is the accelerometer output matrix and is express by $\left[\begin{array}{lll}A_{1} & A_{4} & A_{7}\end{array}\right]^{T}$.

If we measure the system $m$ times, we get $\left(A_{x k}, A_{y k}, A_{z k}, A_{1 k}, A_{4 k}, A_{7 k}\right), k=1, \ldots \ldots, m$ from (20). First consider to fit the first row of $H$ and substitute the measured data into (20), and we get

$$
\left[\begin{array}{c}
A_{11} \\
\vdots \\
A_{1 m}
\end{array}\right]=\left[\begin{array}{cccc}
A_{x 1} & A_{y 1} & A_{z 1} & 1 \\
\vdots & \vdots & \vdots & \vdots \\
A_{x m} & A_{y m} & A_{z m} & 1
\end{array}\right]\left[\begin{array}{c}
\cos \alpha_{1} \cos \beta_{1} \\
\sin \alpha_{1} \cos \beta_{1} \\
\sin \beta_{1} \\
C_{1}
\end{array}\right] .
$$

The solution of the linear equation is the first row of the coupling coefficient matrix. But for that both of the calibrating matrix $A_{I N}$ of the accelerometer input and the accelerometer output matrix $A_{\text {out }}$ have errors, we can not arrive the solution with high precision by using the traditional method. While using TLS, the solution to (21) has the minimum norm to $A_{I N}$ and $A_{\text {out }}$ simultaneously by using TLS. We also can find the TLS solution of the second and third row of $H$ by using the same method. Now we reconstruct the input signal $\left[\begin{array}{lll}A_{x} & A_{y} & A_{z}\end{array}\right]^{T}$ based on the output sig$\operatorname{nal}\left[\begin{array}{lll}A_{1} & A_{4} & A_{7}\end{array}\right]^{T}$.

For that the coupling coefficient matrix $H_{T L S}$ is acquired, there exists an input signal with respect to the measured output signal fulfill (20), namely 


$$
\left[\begin{array}{l}
A_{1} \\
A_{4} \\
A_{7}
\end{array}\right]=H_{T L S}\left[\begin{array}{c}
A_{x} \\
A_{y} \\
A_{z} \\
1
\end{array}\right] .
$$

With the characteristic of the Moore-Penrose generalized inverse matrix , we get

$$
\left[\begin{array}{c}
A_{x} \\
A_{y} \\
A_{z} \\
1
\end{array}\right]=H_{T L S}^{+}\left[\begin{array}{c}
A_{1} \\
A_{4} \\
A_{7}
\end{array}\right],
$$

where $H_{T L S}^{+}$is the generalized inverse matrix with respect to $H_{T L S}$. Using (23), the accelerometer output signal $\left[\begin{array}{lll}A_{x} & A_{y} & A_{z}\end{array}\right]^{T}$ can be reconstructed. Replac$\operatorname{ing}\left[\begin{array}{lll}A_{1} & A_{4} & A_{7}\end{array}\right]^{T}$ by $\left[\begin{array}{lll}A_{x} & A_{y} & A_{z}\end{array}\right]^{T}$ and substituting $\left[\begin{array}{lll}A_{x} & A_{y} & A_{z}\end{array}\right]^{T}$ into (14), we can get the angular acceleration without coupling error.

\section{$5 \quad$ Simulation Results}

After analyzing the static decoupling method to the accelerometer of the NGMIMU system mentioned above using TLS, simulations of the system with different coupling angles are performed respectively in this section. Table 1 and Table 2 illustrate the measured results and the output values of the angular rate after decoupling by using the method in this paper with different sampling numbers, respectively.

In the simulation, the initial conditions in position, velocity, posture angle and angular rate are $x(0)=0 \mathrm{~m}, \quad y(0)=0 \mathrm{~m}, \quad z(0)=0 \mathrm{~m}, v_{x}(0)=0 \mathrm{~m} / \mathrm{s}, \quad v_{y}(0)=0 \mathrm{~m} / \mathrm{s}$, $v_{z}(0)=0 \mathrm{~m} / \mathrm{s}, \alpha_{x}=0 \mathrm{rad}, \quad \alpha_{y}=0 \mathrm{rad}, \alpha_{z}=\pi / 3 \mathrm{rad}, \omega_{x}(0)=0 \mathrm{rad} / \mathrm{s}, \omega_{y}(0)=0 \mathrm{rad} / \mathrm{s}$, $\omega_{z}(0)=0 \mathrm{rad} / \mathrm{s}$ respectively. The accelerometer static bias is $10^{-5} \mathrm{~g}$. The sampling time is $10 \mathrm{~ms}$. Fig. 4 is curve of the decoupling error at different coupling error.

Table 1. Decoupling results when coupling error is 0.001 rad

\begin{tabular}{ccccc}
\hline $\begin{array}{c}\text { Sampling } \\
\text { number N }\end{array}$ & $\begin{array}{c}\text { Real value } \\
\omega_{z}(\mathrm{rad} / \mathrm{s})\end{array}$ & $\begin{array}{c}\text { Before decouping } \\
\omega_{z c}(\mathrm{rad} / \mathrm{s})\end{array}$ & $\begin{array}{c}\text { After decoupling } \\
\omega_{z d c}(\mathrm{rad} / \mathrm{s})\end{array}$ & $\begin{array}{c}\text { Decoupling } \\
\text { error }(\%)\end{array}$ \\
\hline 10 & 0.000499 & 0.000815 & 0.000548 & 9.82 \\
50 & 0.002397 & 0.003683 & 0.002550 & 6.38 \\
100 & 0.004207 & 0.006736 & 0.004452 & 5.82 \\
150 & 0.004987 & 0.008811 & 0.005243 & 5.13 \\
200 & 0.004564 & 0.009722 & 0.004769 & 4.49 \\
300 & 0.000756 & 0.034278 & 0.000801 & 5.95 \\
400 & -0.003784 & 0.039546 & -0.003626 & 3.25 \\
500 & -0.004794 & 0.048781 & -0.004648 & 3.05 \\
\hline
\end{tabular}


Table 2. Decoupling results when coupling error is $0.005 \mathrm{rad}$

\begin{tabular}{ccccc}
\hline $\begin{array}{c}\text { Sampling } \\
\text { number N }\end{array}$ & $\begin{array}{c}\text { Real value } \\
\omega_{z}(\mathrm{rad} / \mathrm{s})\end{array}$ & $\begin{array}{c}\text { Before decouping } \\
\omega_{z c}(\mathrm{rad} / \mathrm{s})\end{array}$ & $\begin{array}{c}\text { After decoupling } \\
\omega_{z d c}(\mathrm{rad} / \mathrm{s})\end{array}$ & $\begin{array}{c}\text { Decoupling } \\
\text { error }(\%)\end{array}$ \\
\hline 10 & 0.000499 & 0.001879 & 0.000574 & 15.03 \\
50 & 0.002397 & 0.008616 & 0.002605 & 8.67 \\
100 & 0.004207 & 0.016490 & 0.004549 & 8.13 \\
150 & 0.004987 & 0.022333 & 0.005385 & 7.98 \\
200 & 0.004564 & 0.028845 & 0.004921 & 7.82 \\
300 & 0.000756 & 0.036292 & 0.000825 & 9.12 \\
400 & -0.003784 & 0.043270 & -0.003563 & 5.84 \\
500 & -0.004794 & 0.056988 & -0.004577 & 4.53 \\
\hline
\end{tabular}

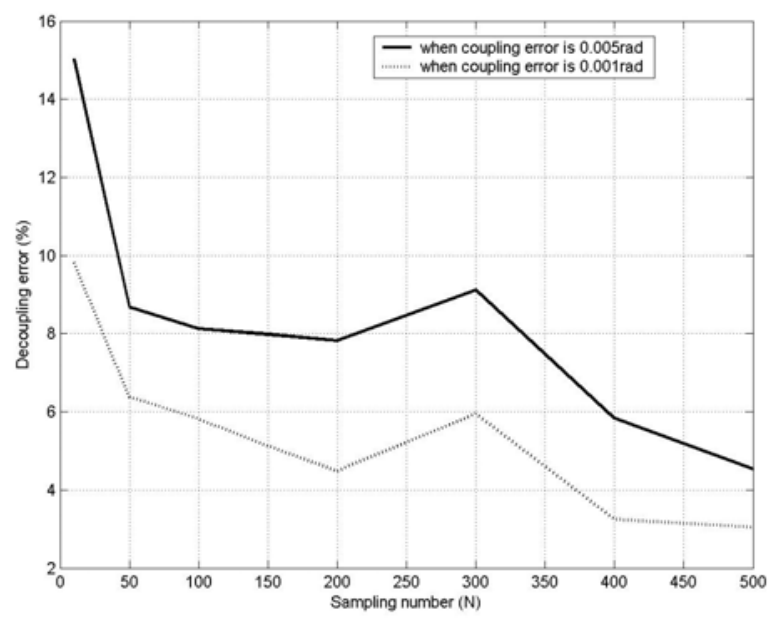

Fig. 4. Curves of decoupling error

From Table 1, Table 2 and Fig. 4, it is obvious that the value of the angular rate before decoupling distorts seriously no matter what the coupling angle is. Under the situation of the same coupling angle, with the increasing of the sampling numbers, the decoupling error ratio $\left(\frac{\left|\omega_{z h}-\omega_{z}\right|}{\left|\omega_{z}\right|} \times 100 \%\right)$ is smaller and smaller. The value of the decoupling error ratio is smaller than $8 \%$ and near to 0 . The increasing estimating precision is due to the increasing sampling points which fit the coupling coefficient matrix correctly. Comparing Tab.1 and Tab.2, it is obvious that the decoupling error ratio in Tab.1 is less than that in Tab.2. The reason is that the larger the coupling angle is, the serious the distortion degree of the accelerometer is. The simulation results show that the static decoupling method can reconstruct the input signal with high precision effectively. 


\section{Conclusions}

The static coupling error is one of the main system errors which seriously affect the estimating precision when the navigation calculation is being carrying on to a NGMIMU system. The decoupling problem can be transform into a problem of solving an equation by changing the system mathematics model with coupling error into a format like $A X=b$. In this paper, the decoupling method by using TLS and MoorePenrose generalized inverse matrix considers not only the error of the accelerometer output but also the error of the input signal for calibrating the system. The method highly improves the estimating precision of the coupling coefficient and the reconstructing precision of the input signal. And it is necessary to be home in on the analysis of the dynamic coupling error with nonlinear characteristic in further research.

Acknowledgments. This work is supported by "the National Natural Science Foundation of China" (Grant No. 60901042 and No. 61171189) and "the Fundamental Research Funds for Central Universities” (Grant No. HIT. NSRIF. 2010105).

\section{References}

1. Schuler, A.R.: Measuring Rotational Motion with Linear Accelerometers. IEEE Trans. on AES 3(3), 465-472 (1967)

2. Merhav, S.J.: A Nongyroscopic Inertial Measurement Unit. J. Guidance 5(3), 227-235 (1982)

3. Tan, C.-W., Park, S.: Design of Gyroscope-free Navigation Systems. In: 2001 Proceedings of Intelligent Transportation Systems, pp. 286-291 (2001)

4. Lee, S.C., Huang, Y.C.: Innovative Estimation Method with Measurement Likelihood for All-accelerometer Type Inertial Navigation System. IEEE Trans. on AES 38(1), 339-346 (2002)

5. Kao, C.F., Chen, T.L.: Design and Analysis of an Orientation Estimation System Using Coplanar Gyro-free Inertial Measurement Unit and Magnetic Sensors. Sensor and Actuators A 144, 251-262 (2008)

6. Wang, J., Wang, Q., Sun, S.: Optimum technology for non-gyro micro inertial measuring unit. Jounal of Harbin Institute of Technology 34(5), 632-635 (2003)

7. Valaee, S., Champagne, B., Kabal, P.: Localization of Wideband Signals Using LeastSquaress and Total Least-Squaress Approaches. IEEE Trans. on Signal Processing 47(5), 1213-1222 (1999)

8. Kubus, D., Kroger, T., Wahl, F.M.: On-line estimation of inertial parameters using a recursive total least-squares approach. In: Proceedings of International Conference on Intelligent Robots and Systems, pp. 3845-3852 (2008)

9. Yang, K., An, J., Bu, X., Sun, G.: Constrained Total Least-Squares Location Algorithm Using Time-Difference-of-Arrival Measurements. IEEE Trans. on Vehicular Technology 59(3), 1558-1562 (2010) 